\title{
EROSION OF FROZEN SULFUR DIOXIDE BY ION BOMBARDMENT: APPLICATIONS TO IO
}

\author{
C. L. Melcher, D. J. LePoire, B. H. Cooper, and T. A. Tombre1lo
}

\section{W. K. Kellogg Radiation Laboratory}

California Iastitute of Technology, Pasadena, California 91125

Abstract. The erosion of frozen $\mathrm{SO}_{2}$ due to bombardment by both light and heavy ions (He and F) was measured for bombarding energies of 0.08 to $1.3 \mathrm{MeV} / \mathrm{amu}$. The number of $\mathrm{SO}_{2}$ molecules ejected from the target per incident ion (i.e., the sputtering yield) was 50 for $1.5 \mathrm{MeV}$ He ions and 7300 for $6 \mathrm{MeV} \mathrm{F}$ ions. Ion bombardment followed by heating produced an oxygen/sulfur residue which was much more stable against subsequent ion bombardment than the initial frozen $\mathrm{SO}_{2}$. The erosion rate of $\mathrm{SO}_{2}$ frost on Jupiter's moon Io depends strongly on the elemental composition and energy spectra of the magnetospheric ion flux which bombards the surface. The combined effects of ion bombardment and heating which produced residues on our target substrates may also occur on Io from magnetospheric ion bombardment and heating by volcanism. Our experimental results compare favorably with a new model of the sputtering process which considers the energy loss of the incident ion to electronic excitation in the target.

\section{INTRODUCTION}

The bombardment of planetary surfaces by the solar wind, solar flare particles, and magnetospheric particles has been suggested as a possible mechanism for modification of these surfaces as well as for the generation of coronae (see, e.g., Matson et al., 1974; Haff et al., 1981; Johnson et al., 1981; Watson, 1981). Measurements of erosion rates of frozen $\mathrm{H}_{2} \mathrm{O}$ due to charged particle bombardment have previously been carried out (Brown et al., 1980; Cooper, 1982). The innermost Galilean moon Io, however, shows evidence of a different kind of ice on its surface, frozen sulfur dioxide. Volcanic gases appear to condense on the cold surface to form an $\mathrm{SO}_{2}$ frost or adsorbate (see e.g., Nelson et al., 1980). This paper describes measurements of the erosion (sputtering) of frozen $\mathrm{SO}_{2}$ due to bombardment by both light and heavy ions (He and F) with energies ranging from 0.08 to $1.3 \mathrm{MeV} / \mathrm{amu}$. Comparison of these data with the theoretical work of Watson and Tombrello (1982a,b) should also provide a reliable basis for calculation of the erosion rate of $\mathrm{SO}_{2}$ by other ions with other energies.

An additional motive behind this work is to investigate the physical processes involved in sputtering. The widely used sputtering theory of Sigmund (1969) predicts negligible erosion due to incident particles with energies exceeding several $\mathrm{keV} / \mathrm{amu}$. In recent years, however, a number of measurements on dielectric materials have shown

Copyright 1982 by the American Geophysical Union.

Paper number 2L1328.

$0094-8276 / 82 / 002 \mathrm{~L}-1328 \$ 3.00$ the existence of an enhanced sputtering effect when insulating targets are bombarded with ions having energies in the $0.1-1.0 \mathrm{MeV} /$ amu range (Biersack and Santner, 1976; Brown et al., 1980; Griffith et al., 1980; Qiu et a1., 1982 ; Diick et a1., 1980;011erhead et a1., 1980). The effect has been observed in diverse materials including CsI, ergosterol, $\mathrm{UF}_{4}, \mathrm{KC} 1, \mathrm{H}_{2} \mathrm{O}, \mathrm{Al}_{2} \mathrm{O}_{3}, \mathrm{LiNb} 0_{3}$, Xe, $\mathrm{SiO}_{2}$, and $\mathrm{CaF}_{2}$. Frozen $\mathrm{SO}_{2}$ also shows this effect and the data presented here are of interest for comparison with the previously studied materials to help in deciding which of several proposed models best describes the enhanced high energy sputtering process. Fluorine was chosen for "he heavy ion bombardment in the present study to enable direct comparison with sputtering data on other materials, especially $\mathrm{UF}_{4}$ and $\mathrm{H}_{2} \mathrm{O}$. In addition, fluorine is close in mass and nuclear charge to oxygen which is a major component of the Jovian magnetospheric ion flux.

\section{EXPERTMENTAL PROCEDURE}

The sputtering yields (number of $\mathrm{SO}_{2}$ molecules removed per incident ion) for $\mathrm{He}$ and $\mathrm{F}$ ions incident on solid $\mathrm{SO}_{2}$ targets were measured for $\mathrm{F}$ bombarding energies ranging from 1.6 to $20 \mathrm{MeV}$ and $1.5 \mathrm{MeV}$ He ions. The frozen $\mathrm{SO}_{2}$ targets (2000-4000 $\AA$ thick) were formed by vapor deposition onto a cold (100K) substrate. This temperature was maintained by flowing liquid helium through an open cycle transfer line on which the substrate was mounted. The substrate consisted of a thick Be disk onto which a $50 \AA$ Au film had been evaporated. The substrate was located in an ultra-high vacuum chamber with a base pressure of 10-9 torr. The apparatus is described in more detail elsewhere (Cooper, 1982; LePoire et al., 1982).

Sputtering yields were obtained by measuring the initial target thickness, bombarding the target with a known dose of energetic ions, and then measuring the final target thickness. Target thicknesses were obtained by backscattering spectrometry of $1.5 \mathrm{MeV}{ }^{4} \mathrm{He}^{+}$ions using a silicon surface barrier detector; the $F$ ion dose was calculated from the number of ions scattered by the Au marker. Three methods of calculating the $\mathrm{SO}_{2}$ thickness from the backscattering spectra were used: the energy shift of the substrate Au peak, the number of $S$ counts, and the number of 0 counts. The sputtering yields calculated using these methods always agreed to within $\pm 15 \%$.

Targets deposited at $100 \mathrm{~K}$ were non-stoichiometric, with 10-25\% more oxygen than expected for pure $\mathrm{SO}_{2}$. The excess 0 was reduced to $5-10 \%$ by raising the temperature of the target substrate to $300 \mathrm{~K}$ during target formation. The backscattering spectra indicated that the targets were free of any other contaminants. 
TABLE 1. SPUTTERING YIELDS

Ion Energy (MeV) $\begin{gathered}\text { Number of } \\ \text { Measurements }\end{gathered} \quad \begin{gathered}\text { Yields* } \\ \text { (molecules/ion) }\end{gathered}$

\begin{tabular}{llll}
\hline $\mathbf{H e}^{+}$ & 1.5 & 6 & $50 \pm 5$ \\
$\mathbf{F}^{+2}$ & 1.6 & 2 & $3515 \pm 320$ \\
$\mathbf{F}^{+3}$ & 6.0 & 2 & $7325 \pm 30$ \\
$\mathbf{F}^{+3}$ & 7.5 & 3 & $6492 \pm 562$ \\
$\mathbf{F}^{+3}$ & 10.0 & 4 & $4647 \pm 261$ \\
$\mathbf{F}^{+4}$ & 15.0 & 8 & $3701 \pm 665$ \\
$\mathbf{F}^{+4}$ & 20.0 & 2 & $2172 \pm 18$ \\
$\mathbf{F}^{+5}$ & 25.0 & 4 & $1632 \pm 141$
\end{tabular}

*The errors listed are one standard deviation when three or more measurements were made; when only two measurements were made, the errors indicate the difference between the two measurements.

\section{RESULTS}

The results of bombarding frozen $\mathrm{SO}_{2}$ with 1.5 $\mathrm{MeV}$ He and 1.6 to $25 \mathrm{MeV} \mathrm{F}$ ions are listed in Table 1. The erosion rates (sputtering yields) for $\mathrm{F}$ ion bombardment of $\mathrm{SO}_{2}$ are shown as a function of bombarding energy in Fig. 1 as filled circles. The dashed line shows the erosion rate for $\mathrm{H}_{2} \mathrm{O}$ ice multiplied by five (from Cooper, 1982). Also shown in Fig. 1 is the stopping power of $19 \mathrm{~F}$ in $\mathrm{SO}_{2}$. The yields, ranging up to $z 7000$ at 6 $\mathrm{MeV}$, are orders of magnitude larger than expected on the basis of Sigmund sputtering theory and are apparently associated with energy loss of the incident ions to electronic processes in the target. The sputtering yield was independent of the ion beam current density up to $0.5 \mu \mathrm{A} / \mathrm{cm}^{2}$ (see LePoire et al., 1982).

An unexpected phenomenon was observed following each He and $\mathrm{F}$ sputtering run when the $\mathrm{SO}_{2}$ was removed from the target by warming the substrate to room temperature. Surprisingly, some $S$ and 0 remained on the Be/Au substrate even at $300^{\circ} \mathrm{K}$. Backscattering analysis showed that this residue was enriched in 0 by a factor of two to three. The residue was typically $\sim 5-10 \%$ of the original target thickness and was located predominantly where the ion beams had hit the target. Areas of the target which had not been bombarded retained almost no residue. The erosion rate of the residue at $10^{\circ} \mathrm{K}$ was about a factor of ten less than that for frozen $\mathrm{SO}_{2}$ at $10^{\circ} \mathrm{K}$ and decreased slightly after prolonged bombardment. Precise measurement of the erosion rate of the residue was not possible due to its rather ill-defined nature and time-dependent composition. Oxygen was preferentially removed from the residue by $F$ ion bombardment.

\section{DISCUSSION}

Charged particle fluxes were measured in the Jovian magnetosphere by the Voyager low energy charged particle (LECP) experiment ( Krimigis et al., 1979). Inside the orbit of Callisto, however, the system was operated in a mode that did not resolve different ion species, and the ion flux data near Io has been interpreted in various ways (see Johnson et al., 1981; Eviatar et a1., 1981). Rather than choose a particular interpretation of LECP flux data, we present in Fig. 2 the erosion rate of frozen $\mathrm{SO}_{2}$ for a wide range of incident ion fluxes. Two simple cases are shown in which the flux is assumed to consist entirely of $1.5 \mathrm{MeV}$ He or $1.6 \mathrm{MeV} \mathrm{F}$ ions. For comparison, the erosion rates for water ice are also shown using the data of Cooper (1982). Although $F$ is not a major component of the magnetospheric particle flux at lo, the erosion rate due to bombardment by 0 is expected to be quite similar, though somewhat lower. Thus, the F results may be taken as an upper bound for the erosion due to 0 ion bombardment.

The erosion rates calculated here reflect bombardment by high energy ions only. In order to obtain the total erosion rate, the sputtering yield must be integrated over the energy spectrum of the incident flux. At lower bombarding energies $(\sim 1 \mathrm{keV} / \mathrm{amu})$ the sputtering yield is expected to be much lower, although it has not yet been measured. On the other hand, the incident flux at lower energies is considerably larger. Eviatar et al. (1981) have argued that the sputtering yield of real planetary surfaces might be considerably lower than that of their analogs in laboratory experiments. If this is true, the erosion rates given here should be considered as upper limits.

Various interpretations of the Voyager LECP data suggest particle fluxes near $1 \mathrm{MeV}$ of $10^{4}$. $106 \mathrm{~cm}^{-2} \mathrm{sec}^{-1}$ in the vicinity of Io (see Krimigis et a1., 1979; Lanzerotti et al., 1982). Although the ion species are not known with certainty in this energy range, large 0 ion fluxes $\left(10^{3}-10^{4}\right.$ $\mathrm{cm}^{-2} \mathrm{sec}^{-1}$ ) are observed at energies above $7 \mathrm{MeV}$ (Vogt et al., 1979; Gehrels et al., 1981), and Lanzerotti et al., (1982) suggest that the flux near $1 \mathrm{MeV}$ may be dominated by heavy ions such as 0 and $S$. Using our $F$ measurements as a guide, an $\mathrm{MeV} O$ ion flux of $10^{4}-10^{6} \mathrm{~cm}^{-2} \mathrm{sec}^{-1}$ would result in an $\mathrm{SO}_{2}$ erosion rate of roughly 1-100 mm per million years. Thus, if the $\mathrm{SO}_{2}$ frost layer is thin (but optically thick), sputtering by magnetospheric ions might expose underlying mate-

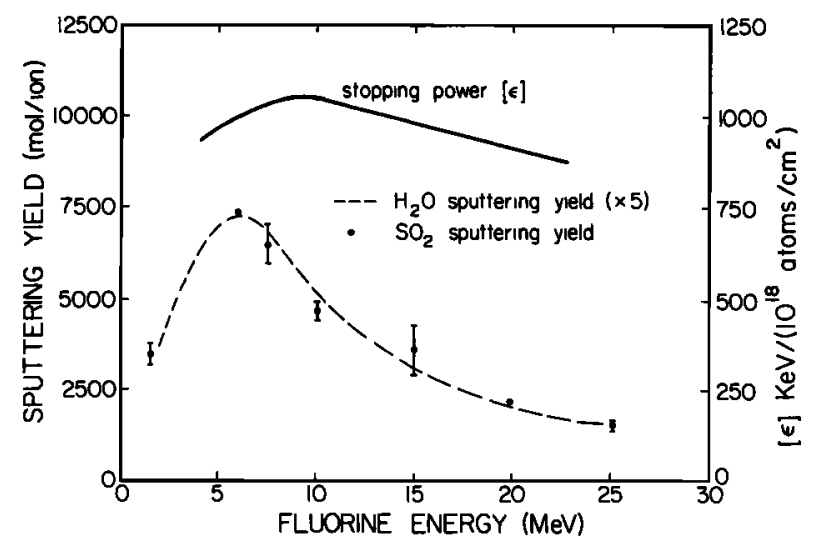

Fig. 1. Sputtering yield of frozen $\mathrm{SO}_{2}$ bombarded by $1.6-25 \mathrm{MeV} \mathrm{F}$ ions. The dashed line is the sputtering yield of frozen $\mathrm{H}_{2} \mathrm{O}$ multiplied by five (from Cooper, 1982). Also shown (solid line) is the stopping power of $19_{\mathrm{F}}$ in $\mathrm{SO}_{2}$. 
rial and thereby alter the color and albedo of those surface regions (if any) which are not resurfaced by deposition from volcanic activity. Note that most of the material that is sputtered by high energy ions is not lost from Io but only redistributed due to the low mean energy ( $s \quad 0.1$ $\mathrm{eV}$ ) of the sputtered particles (R. E. Johnson, private communication) relative to the escape energy for an $\mathrm{SO}_{2}$ molecule $(2.2 \mathrm{eV})$.

Lanzerotti et al. (1982) report measurements of $\mathrm{SO}_{2}$ erosion by bombardment with $1.5 \mathrm{MeV} \mathrm{H}$, He, 0 , and $\mathrm{Ar}$ ions as well as 50 and $750 \mathrm{keV}$ protons. Since these measurements are not sufficient to clearly define the erosion rate as a function of bombarding energy, these authors use an energyloss-squared model to calculate this curve. Although this model provides reasonable results for protons and alphas, it underestimates the sputtering yield for heavier ions and the usefulness of more experimental data becomes apparent. For instance, the energy dependence of the sputtering yield seen in Fig. 1 is more sharply peaked and the yield is larger than predicted by the energy-loss-squared model.

The combined effects of ion bombardment followed by heating which produced residues on our target substrates may also occur on the surface of Io. Such a residue might form on an initially cold surface region that is covered with $\mathrm{SO}_{2}$ frost and bombarded by magnetospheric ions and is later heated by subsurface activity. This process could be a factor in the type of surface material present on Io and also could affect the amount of materlal sputtered from the surface into the atmosphere or plasma torus since the erosion rate of the residue is about 10 times less than for frozen $\mathrm{SO}_{2}$. Also, the composition of the flux of sputtered atoms from the residue is quite different from frozen $\mathrm{SO}_{2}$ in that oxygen is preferentially removed from the residue, leaving a substance which is increas ingly enriched in sul-

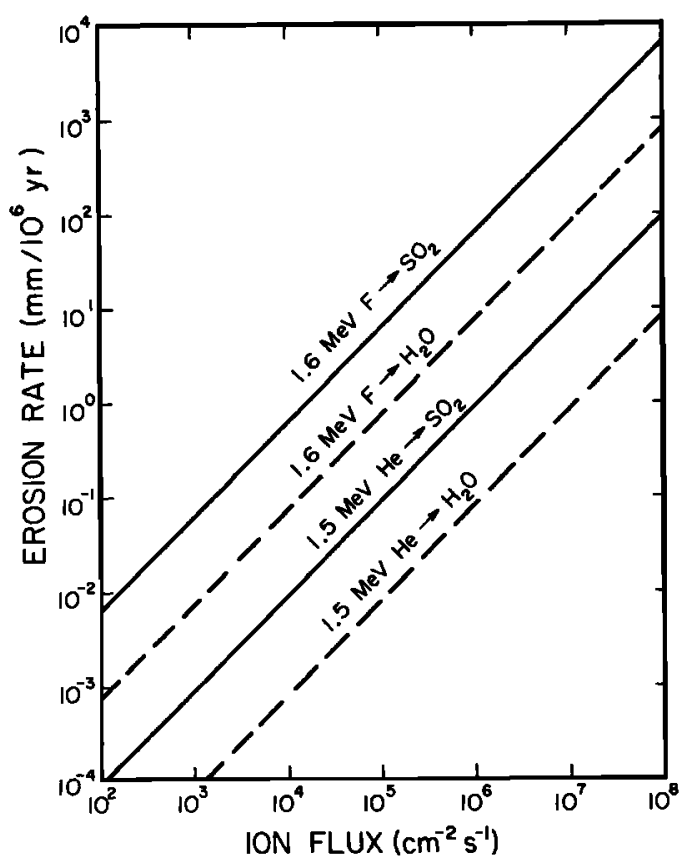

Fig. 2. Erosion rates of frozen $\mathrm{SO}_{2}$ and $\mathrm{H}_{2} \mathrm{O}$ bombarded by $\mathrm{He}$ and $\mathrm{F}$ ions.

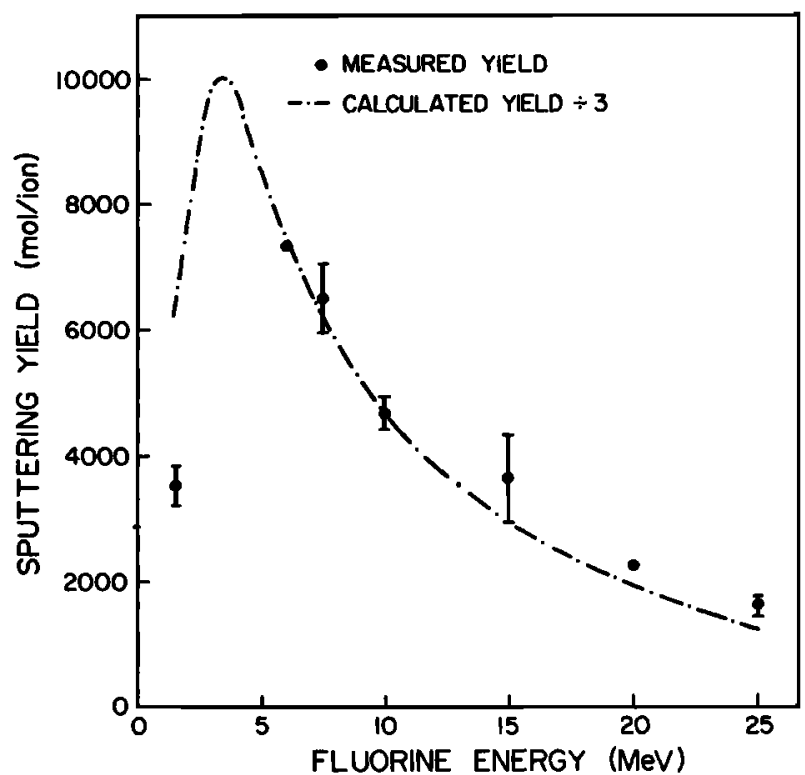

Fig. 3. Comparison of the measured sputtering yield of $\mathrm{F}$ incident on $\mathrm{SO}_{2}$ and the calculated yield from the modified lattice potential model of Watson and Tombre1lo (1982). The yield calculated at energies below the peak are less certain due to the uncertainty in the stopping power.

fur. Such a process could only occur, however, in regions that are heated by subsurface activity but not resurfaced by volcanic deposition.

As can be seen in Fig. 1, the dependence of the sputtering yield on the energy of the bombarding lons is remarkably similar for $\mathrm{SO}_{2}$ and $\mathrm{H}_{2} \mathrm{O}$. In fact, the characteristic shape of this curve is not confined to condensed gas targets but has also been observed in CsI, $\mathrm{UF}_{4}, \mathrm{Al}_{2} \mathrm{O}_{3}$, and $\mathrm{LiNbO}_{3}$ (Hakanson and Sundqvist, 1981; Griffith et al., 1980; Qiu et al., 1982). These data suggest that the same physical process is responsible for the erosion by high energy ion bombardment of a wide range of insulating materials. The process appears not to be due to some special property of frozen gas targets or of the bombarding ions.

Watson and Tombrello (1982a, b) have recently proposed a model which shows considerable promise in explaining the sputtering mechanism. Fig. 3 compares the results of their calculations and the measured yield for $\mathrm{F}$ ions incident on frozen $\mathrm{SO}_{2}$. The calculated yields are a factor of three higher than the measured yields but the similarity in the dependence of projecticle energy is striking. Application of this model to $\mathrm{H}_{2} \mathrm{O}$ ice shows even better agreement with experimental data in that the magnitude as well as the energy dependence of the sputtering yield agrees with measured values to within $20 \%$. Particularly significant is the fact that this model has no free parameters and is not normalized to the data in any way. Only the energy loss rate ( $\mathrm{dE} / \mathrm{dx}$ ) of the incident particles and physical properties of the target such as sublimation energy and electronic band gap are needed in order to predict the sputtering yield. Although its limitations are not yet fully known, this theory has also been tested successfully on other diverse materials including 
$\mathrm{UF}_{4}$ and $\mathrm{SiO}_{2}$ (see Watson and Tombre11o, 1982a). This model promises to be useful in calculating the total integrated sputtering yield on Io once the ion flux composition and energy spectra are known with more confidence and the nature of the various surface materials present there is more clearly defined.

Acknowledgments. We are grateful for discussions with P. K. Haff and C. C. Watson. This work was supported in part by NASA [NAGW-148, NAGW-202] and the NSF [PHY79-23638, CHE81-13273].

\section{REFERENCES}

Biersack, J. P., and E. Santner, Sputtering of potassium chloride by $\mathrm{H}$, He, and $\mathrm{Ar}$ ions, Nuc1. Instru. Meth., 132, 229-235, 1976.

Brown, W. L., W. M. Augustyniak, E. Brody, B. Cooper, L. J. Lanzerotti, A. Ramirez, R. Evatt, and R. E. Johnson, Energy dependence of the erosion of $\mathrm{H}_{2} \mathrm{O}$ ice films by $\mathrm{H}$ and $\mathrm{He}$ ions, Nucl. Instru. Meth., 170, 321-325, 1980.

Cooper, B. H., Erosion of ice films by energetic ions. Ph.D. thesis, Calif. Inst. of Technol., Pasadena, 1982.

Duck, P., W. Treu, H. Frölich, W. Galster, and H. Voit, Desorption of organic compounds from solid surfaces by bombardment with heavy ions from a tandem accelerator, Surface Sci., 95, $603,1980$.

Eviatar, A., G. L. Siscoe, T. V. Johnson, and D. L. Matson, Effects of Io ejecta on Europa, Icarus, 47, 75-83, 1981.

Gehrels, N., E. C. Stone, and J. H. Trainor, Energetic oxygen and sulfur in the Jovian magnetosphere, J. Geophys. Res., 86, 8906, 1981.

Griffith, J. E., R. A. Weller, L. E. Seiberling, and T. A. Tombre11o, Sputtering of Uranium tetrafluoride in the electronic stopping regime, Rad. Effects, 51, 223, 1980.

Haff, P. K., C. C. Watson, and Yuk L. Yung, Sputter ejection of matter from Io, J. Geophys. Res., 86, 6933-6938, 1981.

Hakkansson, P., E. Jayansinghe, A. Johansson, I. Kamensky, and B. Sundqvist, Charge-state dependence of desorption of biomolecules induced by fast heavy ions, Phys. Rev. Lett., 47, 1127, 1981 .

Johnson, R. E., L. J. Lanzerotti, W. L. Brown, and T. P. Armstrong, Erosion of Galilean satellite surfaces by Jovian magnetospheric particles, Science, 212, 1027-1030, 1979.

Lanzerotti, L., W. L. Brown, W. Augustyniak, R. E. Johnson, and T. P. Armstrong, Laboratory studies of charged particle erosion of $\mathrm{SO}_{2}$ ice and applications to the frosts of Io, Astrophys. J., 259, 920-929, 1982.

LePoire, D. J., B. H. Cooper, C. L. Melcher, and T. A. Tombrello, Sputtering of $\mathrm{SO}_{2}$ by high energy ions, Rad. Effects, (submitted) 1982.

Matson, D. L., T. V. Johnson, and F. P. Fanale, Sodium D-1ine emission from Io: Sputtering and resonant scattering hypothesis, Astrophys. J., 192, L43, 1974.

Ne'lson, R. M., A. L. Lane, D. L. Matson, F. P. Fanale, D. B. Nash, and T. V. Johnson, Io: Longitudinal distribution of sulfur dioxide frost, Science, 210, 784, 1980.

Ollerhead, R. W., J. Bottiger, J. A. Davies, J. L. Ecuyer, H. K. Haugen, and N. Matsunami, Evidence for a thermal spike mechansim in the erosion of frozen xenon, Rad. Effects, 49, 203-212, 1980.

Qiu, Yuanxun, J. E. Griffith, and T. A. Tombre110, Sputtering of $\mathrm{Al}_{2} \mathrm{O}_{3}$ and $\mathrm{LiNbO}_{3}$ in the electronic stopping region, Rad. Effects, in press, 1982.

Sigmund, P., Theory of Sputtering, I. Sputtering yield of amorphous and polycrystalline targets, Phys. Rev., 184, 383-416, 1969.

Vogt, R. E., W. R. Cook, A. C. Cummings, T. L. Garrard, N. Gehrels, E. C. Stone, J. H. Trainor, A. W. Schardt, T. Conlon, N. Lal, and F. M. McDonald, Voyager 1: Energetic ions and electrons in the Jovian magnetosphere, Science, 204, 1003, 1979.

Watson, c. C., The sputter-generation of planetary coronae: Galilean satellites of Jupiter, Proc. Lunar Planet. Sci. Conf. 12th, p. 1569-1583, 1981.

Watson, C. C., and T. A. Tombrello, Enhanced ionerosion of planetary surfaces, in Lunar and Planetary Science XIII, p. 845-846, Lunar and Planetary Science Institute, Houston, 1982a.

Watson, C. C. and T. A. Tombrello, A modified lattice potential model of enhanced ion erosion, to be published (1982b).

(Received April 29, 1982;

accepted August 24, 1982.) 